\title{
Proteasome activator PA28 $\gamma$ stimulates degradation of GSK3-phosphorylated insulin transcription activator MAFA
}

\author{
Kenichi Kanai, Shinsaku Aramata, Sayo Katakami, Kunio Yasuda and Kohsuke Kataoka \\ Laboratory of Molecular and Developmental Biology, Graduate School of Biological Science, Nara Institute of Science and Technology, 8916-5 Takayama-cho, Ikoma 630-0192, Japan \\ (Correspondence should be addressed to K Kataoka; Email: kkataoka@bs.naist.jp)
}

(K Kanai is now at Department of Tissue Morphogenesis, Max-Planck Institute for Molecular Biomedicine, Roentgenstrasse 20, 48149 Muenster, Germany)

\begin{abstract}
MAFA is a member of the MAF family of basic leucine zipper transcription factors and is a critical regulator of insulin gene expression and islet $\beta$-cell function. To be degraded by the proteasome, MAFA must be phosphorylated by GSK3 and MAP kinases at multiple serine and threonine residues (Ser49, Thr53, Thr57, Ser61, and Ser65) within its amino-terminal domain. In this study, we report that MAFA degradation is stimulated by PA28 $\gamma$ (REG $\gamma$ and PSME3), a member of a family of proteasome activators that bind and activate the $20 \mathrm{~S}$ proteasome. To date, only a few PA28 $\gamma$-proteasome pathway substrates have been identified, including steroid receptor coactivator 3 (SRC3) and the cell cycle inhibitor p21 (CIP1). PA28 $\gamma$ binds to MAFA, induces its proteasomal degradation, and thereby attenuates MAFA-driven transcriptional activation of the insulin promoter. Co-expression of GSK3 enhanced the PA28 $\gamma$-mediated degradation of MAFA, but mutants that contained alanine substitutions at the MAFA phosphorylation sites did not bind PA28 $\gamma$ and were resistant to degradation. We also found that a PA28 $\gamma$ mutant (N151Y) that did not stimulate p21 degradation enhanced MAFA degradation, and another mutant (K188D) that promoted greater p21 degradation did not enhance MAFA degradation. These results suggest that PA28 $\gamma$ stimulates MAFA degradation through a novel molecular mechanism that is distinct from that for the degradation of $\mathrm{p} 21$.
\end{abstract}

Journal of Molecular Endocrinology (2011) 47, 119-127

\section{Introduction}

MAFA is a member of the MAF family of basic leucine zipper transcription factors. In avians, MAFA/L-MAF has been identified as a neuroretina-specific transcript (Benkhelifa et al. 1998) or a transcription factor that binds to the lens-specific enhancer in crystallin genes (Ogino \& Yasuda 1998). Gain- and loss-of-function experiments in the developing chicken embryo revealed that MAFA/L-MAF is a key regulator of lens development (Reza et al. 2002).

In mammals, MAFA has been identified as a transactivator of the insulin gene that binds to a critical cis-regulatory element within the promoter termed C1/RIPE3b (Kataoka et al. 2002, Olbrot et al. 2002, Kajihara et al. 2003, Matsuoka et al. 2003). MAFA is exclusively expressed in $\beta$-cells in the pancreas (Kataoka et al. 2004, Zhang et al. 2005, Nishimura et al. 2006) and plays a critical role in establishing $\beta$-cell-specific expression of the insulin gene together with the other $\beta$-cell-enriched transcription factors, PDX1 and BETA2/NEUROD (Aramata et al. 2005, Docherty et al. 2005, Kaneto et al. 2005). MAFA also regulates a set of $\beta$-cell-specific genes, including glucose transporter 2 (glut2) and granuphilin (Zhang et al. 2005, Kato et al. 2006, Wang et al. 2007). In Mafa knockout mice, islets are normal at birth, but $\beta$-cells exhibit a deficiency in glucose-stimulated insulin secretion over time and show progressive degeneration by $8-12$ weeks of age (Zhang et al. 2005, Artner et al. 2007, Nishimura et al. 2008). These observations indicate that MAFA regulates the maturation, functional maintenance, and survival of $\beta$-cells. Recently, it was also shown that MAFA regulates intra-thymic expression of insulin and affects susceptibility to type I diabetes (Noso et al. 2010).

Previous studies have demonstrated that phosphorylation plays critical roles in the regulation of MAFA activity. The amino-terminal domain of MAFA is first phosphorylated by an unidentified priming kinase at Ser65 and then sequentially by GSK3 at Ser61, Thr57, Thr53, and Ser49 (Han et al. 2007, Rocques et al. 2007). Thr57 and Ser65 can also be phosphorylated by ERK and/or p38 MAP kinase (Benkhelifa et al. 2001, Ochi et al. 2003, Sii-Felice et al. 2005). Phosphorylation at these sites is required for proteasomal degradation of MAFA protein (Ochi et al. 2003, Han et al. 2007, Rocques et al. 2007), and mutations at these phosphorylation sites influence the transforming and differentiation-inducing activities of MAFA (Benkhelifa et al. 2001, Nishizawa et al. 2003, Ochi et al. 2003, Pouponnot et al. 2006, Rocques et al. 2007). However, the molecular mechanism by which phosphorylated MAFA is degraded is unknown.

DOI: 10.1530/JME-11-0044 Online version via http://www.endocrinology-journals.org 
PA28 $\gamma$ (REG $\gamma$ and PSME3) is a member of a family of proteasome activators that bind the $20 \mathrm{~S}$ subunit of the proteasome (Dubiel et al. 1992, Ma et al. 1992). PA28 $\gamma$ is predominantly localized within the nucleus and forms homoheptamers, whereas PA28 $\alpha$ and PA28 $\beta$, other members of this family, form heteroheptamers in the cytoplasm (Realini et al. 1997, Wojcik et al. 1998). Biochemical and crystal structural analyses of PA28 family members have suggested that PA28 heptamers associate with both ends of the $20 \mathrm{~S}$ proteasome like 'caps' through interactions between the proteasome and the carboxy-terminal tail of each monomer. On these interactions, the 'activation loop' of PA28 induces a conformational change of the 20S proteasome subunit and stimulates the protease activity (Whitby et al. 2000, Forster et al. 2005). Experiments using peptide substrates revealed that PA28 $\alpha$ and PA28 $\beta$ stimulate three types of $20 \mathrm{~S}$ proteasome catalytic activities: trypsin-like, chymotrypsin-like, and post-glutamyl peptidyl hydrolyzing activities. In contrast, PA28 $\gamma$ stimulates only trypsin-like activity (Realini et al. 1997).

To date, only a few protein substrates of the PA28 $\gamma$-proteasome pathway have been identified. These substrates include steroid receptor coactivator 3 (SRC3), hepatitis C virus core protein, the tumor suppressor p53, and the cell cycle inhibitors p21 (CIP1/WAF1), p16 (INK4A), and p19 (ARF) (Moriishi et al. 2003, Li et al. 2006, 2007, Chen et al. 2007, Zhang \& Zhang 2008). PA28 $\gamma$ directly binds to SRC3 and p21 and promotes their proteasomal degradation independent of poly-ubiquitination and the $19 \mathrm{~S}$ subunit. In contrast, PA28 $\gamma$ stimulates the degradation of p53 by enhancing its ubiquitination by MDM2. These observations suggest that PA28 $\gamma$ is a multi-functional protein involved in the degradation of a subset of nuclear proteins.

In this study, we demonstrate that degradation of MAFA is stimulated by PA28 $\gamma$. PA28 $\gamma$ bound to MAFA and induced its proteasomal degradation in a phosphorylation-dependent manner. In addition, PA28 $\gamma$ attenuated MAFA-driven transcriptional activation of the insulin promoter. Using well-characterized PA28 $\gamma$ mutants (N151Y and K188D) (Zhang et al. 1998, Li et al. 2001), we found that PA28 $\gamma$ stimulated degradation of MAFA and p21 through distinct molecular mechanisms.

\section{Materials and methods}

\section{Plasmids}

The mammalian expression vector for hemagglutinin (HA)-tagged mouse Mafa (pHygEF2/HA-m-MafA) and its derivatives (IRES-EGFP fusions and amino acid substitution mutants) were described previously (Han et al. 2007).

To construct the expression vectors for the PA28 $\alpha$, PA28 $\beta$, and PA28 $\gamma$ FLAG epitope fusion proteins

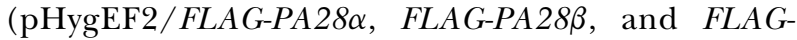
$P A 28 \gamma)$, cDNA fragments containing the entire open frame of mouse PA28 $\alpha$, PA28 $\beta$, and PA28 $\gamma$ were amplified from total RNA isolated from MIN6 mouse insulinoma cells by reverse transcriptase (RT)-PCR using the following primers: Pa28 $\alpha$, $5^{\prime}$-AGAACTAGTATGGCCACACTGAGGGTCCATCCC-3' and $5^{\prime}$-AGAGCGGCCGCTCAATAGATCATTCCCTTGGTTT-3'; Pa28ß, $5^{\prime}$-AGAACTAGTATGGCCAAGCCTTGTGGGGTCCG-3 ${ }^{\prime}$ and $5^{\prime}$-AGAGCGGCCGCTCAGTACATCGA-

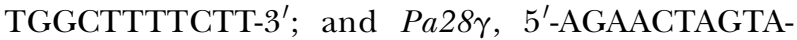
TGGCCTCGTTGCTGAAGGTGG- ${ }^{\prime}$ and $5^{\prime}$-AGAGCGGCCGCTAGTACAGTGTCTCTGCATTG- $3^{\prime}$. The amplified fragments were cloned into the pHygEF2/FLAGSUMO-2 plasmid (Kanai et al. 2010) by replacing the SpeI-NotI fragment.

To construct the FLAG-tagged GSK3 $\beta$ expression vector (pHygEF2/FLAG-GSK3 $\beta$ ), the mouse GSK3 $\beta$ open reading frame was amplified by RT-PCR from mouse liver total RNA using the following primers: 5'-ACTAGTACCATGGATTACAAGGATGACGACGATAAGGGAGGCCTCATGTCGGGGCGACCGAGAACC-3 ${ }^{\prime}$ and $5^{\prime}$-GGCACTCAAGTAAGTGCTGG-3'. The amplified fragment was inserted into the pCRII-TOPO plasmid (Invitrogen) by TA cloning. The SpeI-NotI fragment was excised from the resulting plasmid and inserted into the pHygEF2 vector following digestion with XbaI and NotI.

All of the PA28 $\gamma$ (N151Y and K188D) and GSK3 $\beta$ (S9A and K85R) point mutants were generated by site-directed overhang extension PCR mutagenesis (Ho et al. 1989).

\section{Transfection, immunoprecipitation, and immunoblotting}

NIH3T3 or 293T cells grown in 24-well plates were transfected with a total of $0 \cdot 8 \mu \mathrm{g}$ plasmid using $2 \mu \mathrm{l}$ lipofectamine 2000 reagent (Invitrogen). In1024 cells were transfected with a total of $1 \cdot 6 \mu \mathrm{g}$ plasmid using $4 \mu \mathrm{l}$ lipofectamine 2000 (Invitrogen). After $24 \mathrm{~h}$ of transfection, whole cell extracts were prepared by the direct addition of $4 \times$ SDS sample buffer $(200 \mathrm{mM}$ Tris- $\mathrm{HCl}$ (pH 6.8), 8\% SDS, $400 \mathrm{mM}$ dithiothreitol, $0 \cdot 2 \%$ bromophenol blue, and $40 \%$ glycerol) and then subjected to immunoblot analysis as described previously (Kataoka et al. 2002).

For immunoprecipitation, HeLa cells grown in 6-well plates were transfected with a total of $3 \cdot 2 \mu \mathrm{g}$ plasmid using $8 \mu \mathrm{l}$ lipofectamine 2000 (Invitrogen). Whole cell extracts were prepared by cell lysis in $800 \mu$ l NETN buffer $(150 \mathrm{mM}$ $\mathrm{NaCl}, 1 \mathrm{mM}$ EDTA, $10 \mathrm{mM}$ Tris- $\mathrm{HCl}$, pH $7 \cdot 5$, and $0 \cdot 1 \%$ 
NP-40) containing a protease inhibitor cocktail (Nacarai tesque, Kyoto, Japan), sonicated for $20 \mathrm{~s}$, and then centrifuged. Aliquots of the extracts were subjected to immunoprecipitation using anti-HA-agarose (Roche) and then examined by immunoblot analysis. The antibodies used were as follows: anti-HA (MBL, Nagoya, Japan), anti-FLAG (M2, Sigma), anti-GFP (Clontech, Mountain View, CA, USA), anti-TF-IID (TATA-binding protein (TBP) SI-1; Santa Cruz Biotechnology, Santa Cruz, CA, USA), anti-c-Maf (M-153, Santa Cruz), and anti-PA28 (PW8190; Enzo Life Sciences, Plymouth Meeting, PA, USA). MG132, epoxomicin, lactacystin (Merck), SB216763 (Tocris Bioscience, Ellsville, MO, USA), and cycloheximide (CHX, Sigma) were obtained commercially.

\section{RNAi}

NIT-1 cells grown in 24-well plates were transfected with 15 pmol siRNA using PrimaPort (Credia Japan, Kyoto, Japan) and were subjected to a second round of transfection $24 \mathrm{~h}$ later. A synthetic siRNA targeting mouse $\mathrm{Pa} 28 \gamma$ was purchased from Invitrogen (Psme3-MSS208070).
The pEF3-GFPBsr-U6 plasmid to express short-hairpin RNA (shRNA) was designed to clone a double synthetic oligonucleotide into the U6 small nuclear RNA promoter using BseRI-BglII restriction sites. The oligonucleotides used for silencing human PA28 $\gamma$ were as follows: 5'-GAACCAAGGTGTTTGTGAttcaagagaTCACAAACACCTTGGTTCCttttt- $3^{\prime}$ and $5^{\prime}$-GATCaaaaaGGAACCAAGGTGTTTGTGAtctcttgaaTCACAAACACCTTGGTTCca-3'

\section{Luciferase assay}

The luciferase reporter plasmid (pGL4/h-ins-p) was constructed by inserting a KpnI-HindIII fragment of the pGL2-based h-ins-p-luc plasmid (Kataoka et al. 2002) into pGL4.10 (Promega). pEF-Rluc were described previously (Kataoka et al. 2001). NIH3T3 cells grown in 24-well plates were transfected with a total of $0.8 \mu \mathrm{g}$ plasmid DNA $(0.05 \mu \mathrm{g}$ pGL4/h-ins-p, $0.05 \mu \mathrm{g}$ pEF-Rluc, and $0.7 \mu \mathrm{g}$ of the expression plasmids) using lipofectamine 2000 (Invitrogen). Cells were harvested $24 \mathrm{~h}$ after transfection, and the firefly and Renilla luciferase activities were measured using the Dual Luciferase
A

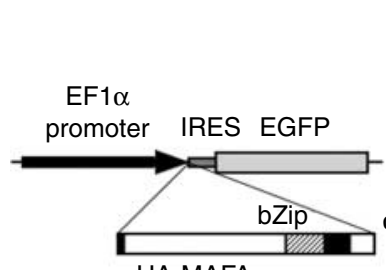

HA-MAFA

D

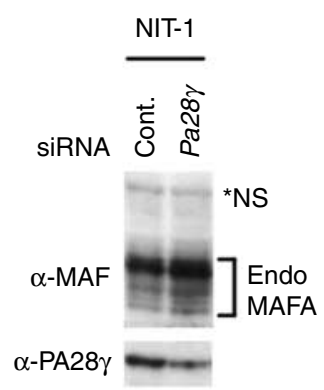

B

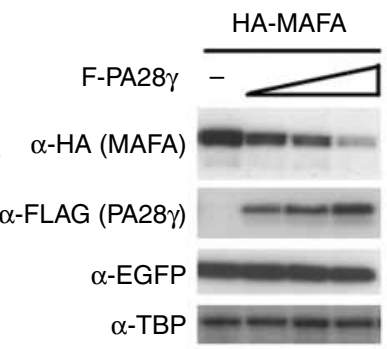

E

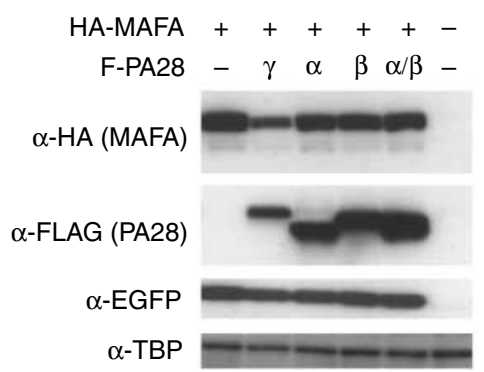

Figure 1 PA28 $\gamma$ decreased the amount of MAFA protein. (A) Schematic structure of the pHygEF2/HA-m-MafA-IRES-EGFP expression plasmid. EF1 $\alpha$, elongation factor $1 \alpha$; IRES, internal ribosome entry site; EGFP, enhanced green fluorescent protein; bZip, basic leucine zipper. (B) NIH3T3 cells were transfected with a constant amount $(0.2 \mu \mathrm{g})$ of pHygEF2/HA-m-MafA-IRES-EGFP and an increasing amount $(0.2,0.4$, and $0.6 \mu \mathrm{g})$ of the FLAG-tagged PA28 $\gamma(\mathrm{F}-\mathrm{PA} 28 \gamma)$ expression plasmid. Total cell extracts were analyzed by immunoblot using anti-HA, anti-FLAG, and anti-EGFP antibodies. TATA-binding protein (TBP) was used as a loading control. (C) Knockdown of PA28 $\gamma$. 293T cells were transfected with expression vectors for HA-MAFA (0.4 $\mu \mathrm{g})$ and Pa28 $\gamma$-targeted shRNA (0.4 $\mu \mathrm{g})$. Cell extracts were analyzed for HA-MAFA and endogenous PA28 $\gamma$. (D) The insulinoma-derived cell line NIT-1 was transfected with Pa28 $\gamma$-targeted siRNA. Endogenous MAFA and PA28 $\gamma$ proteins were analyzed by immunoblot. An asterisk indicates non-specific (NS) cross-reacting material serving as a loading control. (E) NIH3T3 cells were transfected with $0.2 \mu \mathrm{g} H A-m-M a f A-I R E S-E G F P$ expression plasmid together with $0.6 \mu \mathrm{g}$ expression plasmids for F-PA28 $\gamma$, F-PA28 $\alpha$, F-PA28 $\beta$, or F-PA28 $\alpha$ plus F-PA28 $\beta$ as indicated. Total cell extracts were immunoblotted with the indicated antibodies. 


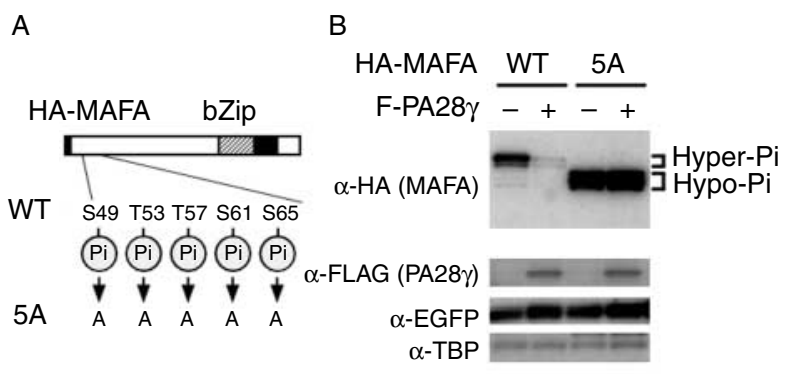

C

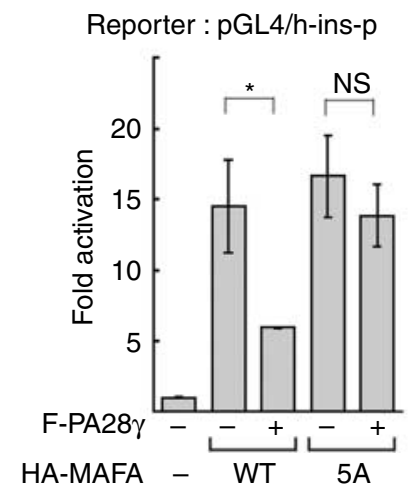

Figure 2 PA28 $\gamma$-induced decrease of phosphorylated MAFA. (A) Schematic structure of wild-type (WT) HA-MAFA and the 5A mutant. (B) Wild-type (WT) HA-MAFA or the 5A mutant fused to IRES-EGFP was expressed in NIH3T3 cells together with or without expression plasmid for F-PA28 $\gamma$. (C) Luciferase assay. A luciferase reporter plasmid driven by the human insulin promoter (pGL4/h-ins- $p$ ) and the indicated combinations of expression plasmids for F-PA28 $\gamma(0.5 \mu \mathrm{g})$ and HA-MAFA (WT or $5 A)(0.2 \mu \mathrm{g})$ were co-transfected into NIH3T3 cells. The luciferase activity was measured and was expressed as fold increase over the luciferase activity in cells that received an empty expression plasmid. Statistical significance was calculated using ANOVA $\left({ }^{\star} P<0 \cdot 05\right)$. NS, not significant.

Assay System (Promega). Data represent the average \pm s.E.M. of two independent experiments. Statistical significance was calculated using ANOVA $(P<0 \cdot 05)$.

\section{Results}

\section{PA28 $\gamma$ reduces the amount of MAFA protein}

To test the possible involvement of PA28 $\gamma$ in the regulation of the amounts of MAFA protein, an expression plasmid for HA-tagged full-length MAFA (HA-MAFA) fused to IRES-EGFP under the control of the constitutively active EF1 $\alpha$ promoter was used (Fig. 1A). We transfected NIH3T3 cells with a constant amount of this plasmid together with an increasing amount of an expression plasmid for FLAG-tagged PA28 $\gamma$ (F-PA28 $\gamma$ ). NIH3T3 was used as a recipient cell for this assay because HA-MAFA protein is relatively stable in this cell line (Han et al. 2007, see also Fig. 3C).
Cell extracts were prepared, and the expression levels of the transgenes were analyzed by immunoblotting with anti-HA and anti-FLAG antibodies. Expression of EGFP was also analyzed to normalize the transfection efficiency, and TBP was monitored as a loading control. The amount of HA-MAFA was reduced by F-PA28 $\gamma$ co-expression in a dose-dependent manner (Fig. 1B).

To examine the role of endogenous PA28 $\gamma$ in the regulation of MAFA protein levels, we used 293T cells because HA-MAFA protein is relatively unstable in this cell line (see Fig. 3D). 293T cells were transfected with an expression plasmid for HA-MAFA together with the shRNA expression vector designed to target PA28 $\gamma$. Immunoblot analysis showed that the amount of MAFA was increased by the knockdown of PA28 $\gamma$ (Fig. 1C).

The effect of PA28 $\gamma$ knockdown on the amount of endogenous MAFA protein was examined. An siRNA designed to target PA28 $\gamma$ was transfected into a $\beta$-cellderived cell line NIT-1 in which endogenous MAFA protein was expressed. Immunoblot analysis showed that endogenous PA28 $\gamma$ was reduced in cells transfected with Pa28 $\gamma$-siRNA and that endogenous MAFA protein was increased in these cells (Fig. 1D). These results indicated that PA28 $\gamma$ might negatively regulate the amount of MAFA protein by inducing degradation.

Then, the effect of PA28 $\alpha$ and PA28 $\beta$, other PA28 family members, on MAFA reduction was determined. PA28 $\gamma$ predominantly localizes in the nuclei and forms a homoheptamer, whereas PA28 $\alpha$ and PA28 $\beta$ localize in the cytoplasm and form heteromeric hexamers (Realini et al. 1997, Wojcik et al. 1998). As shown in Fig. 1E, PA28 $\alpha$ or PA28 $\beta$ alone or in combination did not significantly influence the amount of HA-MAFA.

\section{Phosphorylation of MAFA is required for PA28 $\gamma$-mediated reduction}

Previous studies have shown that MAFA degradation is dependent on phosphorylation at multiple serine and threonine residues (Ser49, Thr53, Thr57, Ser61, and Ser65) in the amino-terminal domain (Han et al. 2007, Rocques et al. 2007). To investigate the role of these phosphorylation sites in MAFA downregulation by PA28 $\gamma$, a MAFA substitution mutant (5A) in which these serine and threonine residues were replaced by alanine residues was examined (Fig. 2A). As we previously observed (Han et al. 2007), the electrophoretic mobility of the 5A mutant protein was higher than that of wild-type (WT) MAFA due to the absence of phosphorylation at the mutated sites (Fig. 2B). In contrast to WT MAFA, the 5A mutant was resistant to PA28 $\gamma$-induced reduction. These results suggested that phosphorylation at these amino acid residues is required for the PA28 $\gamma$-mediated reduction of the amount of MAFA.

To determine whether PA28 $\gamma$ affects the transcriptional activity of MAFA, a luciferase reporter gene driven 
by the human insulin promoter was co-transfected into NIH3T3 cells together with expression plasmids for HA-MAFA and F-PA28 $\gamma$ alone or in combination (Fig. 2C). As previously demonstrated, both WT MAFA and the 5A mutant stimulated reporter activity to a similar degree (Han et al. 2007). Co-expression of F-PA28r reduced transcriptional activation by WT MAFA, but not by the 5A mutant. These results suggested that PA28 $\gamma$ suppressed the transcriptional activity of MAFA by reducing the amount of protein.

\section{PA28 $\gamma$ enhances proteasomal degradation of MAFA}

To determine whether PA28 $\gamma$ reduces the amount of MAFA by enhancing its degradation, NIH3T3 cells were treated with the proteasome inhibitors MG132, epoxomicin, or lactacystin. These drugs blocked the effect of PA28 $\gamma$ on MAFA (Fig. 3A). In 293T cells, MG132 treatment increased the amount of MAFA protein (Fig. 3B). Knockdown of PA28 $\gamma$ resulted in an accumulation of MAFA, and MG132 did not induce further increase in MAFA in these cells. These results indicated that PA28 $\gamma$ promoted the degradation of MAFA by way of the proteasome.

The degradation rates of MAFA in the absence or presence of F-PA28 $\gamma$ were measured. Nascent protein synthesis was blocked in transfected cells using CHX treatment and the amount of HA-MAFA was measured by immunoblotting. WT HA-MAFA disappeared more rapidly in the presence of F-PA28 $\gamma$ than in its absence (Fig. 3C). The phosphorylation-deficient MAFA mutant $(5 \mathrm{~A})$ was stable, and its degradation rate was

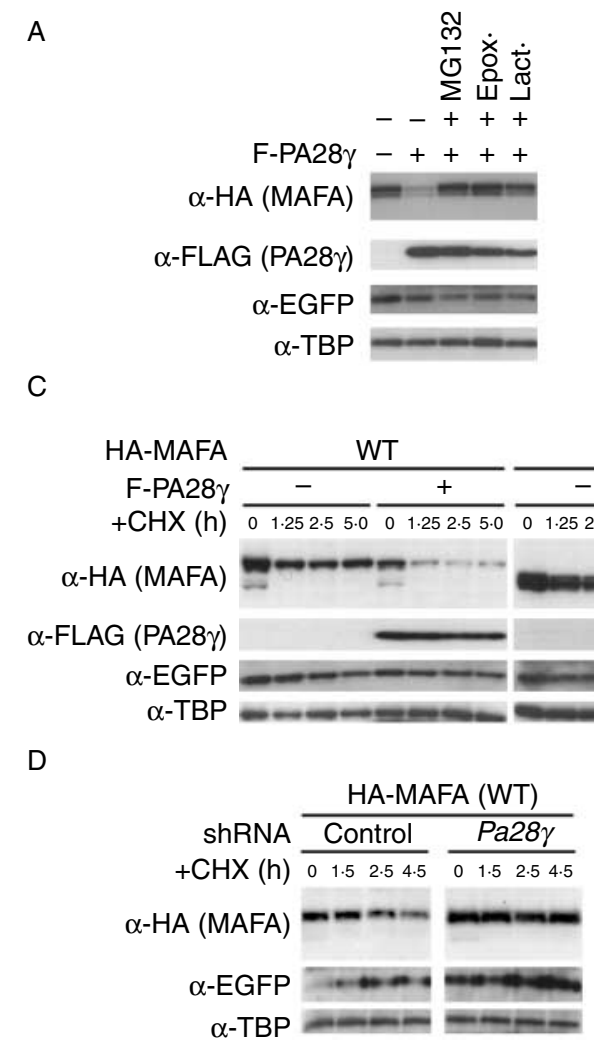

B
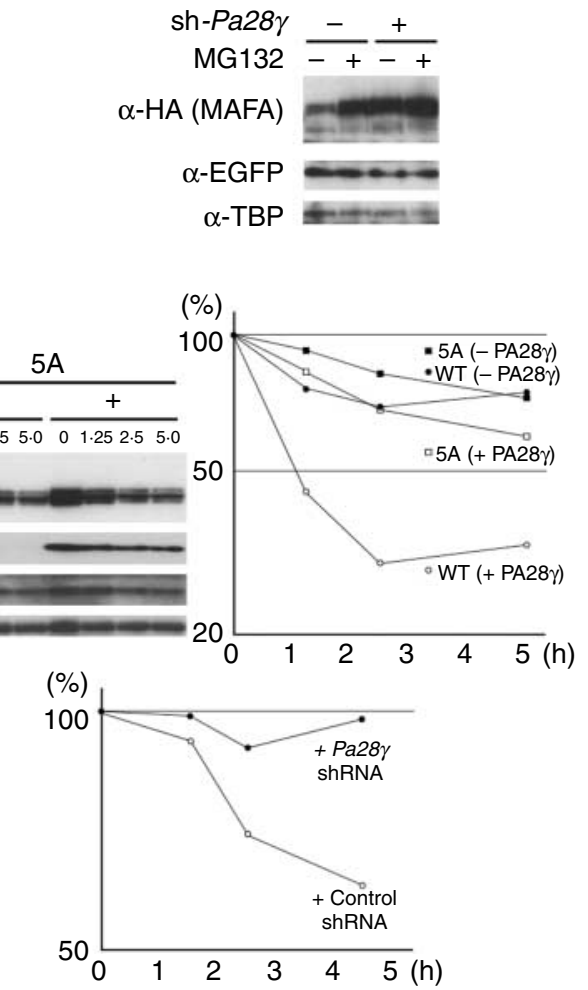

Figure 3 PA28 $\gamma$-induced, phosphorylation-dependent proteasomal degradation of MAFA. (A) NIH3T3 cells transfected with expression vectors for HA-m-MafA-IRES-EGFP and F-PA28 $\gamma$ were treated with the proteasome inhibitors MG132 (5 $\mu \mathrm{M})$, epoxomicin (Epox; $5 \mu \mathrm{M})$, or lactacystin (Lact; $20 \mu \mathrm{M}$ ) for $15 \mathrm{~h}$. Cell extracts were examined by immunoblot analysis using the indicated antibodies. (B) 293T cells transfected with expression vectors for HA-MAFA $(0.4 \mu \mathrm{g})$ and Pa28 $\gamma$-targeted shRNA $(0.4 \mu \mathrm{g})$ were treated with MG132 $(5 \mu \mathrm{M})$ for $15 \mathrm{~h}$. Cell extracts were examined by immunoblot analysis using the indicated antibodies. (C) NIH3T3 cells were transfected with wild-type (WT) HA-MAFA or the 5A mutant fused to IRES-EGFP together with or without the F-PA28 $\gamma$ expression vector. Cells were then treated with $\mathrm{CHX}$ for the indicated times, and the cell extract was examined by immunoblot analysis using the indicated antibodies (left panels). The bands in the anti-HA blot were quantified using ImageJ software and were plotted as a percentage of the initial band intensity of each experiment (right panel). (D) 293T cells were transfected with wild-type (WT) HA-MAFA fused to IRES-EGFP together with control or Pa28 $\gamma$-targeting shRNA expression vector. Cells were treated with $\mathrm{CHX}$ for the indicated times. The cell extracts were examined by immunoblot analysis using the indicated antibodies (left panels). The bands in the anti-HA blot were plotted as a percentage of the initial band intensity of each experiment (right panel). 


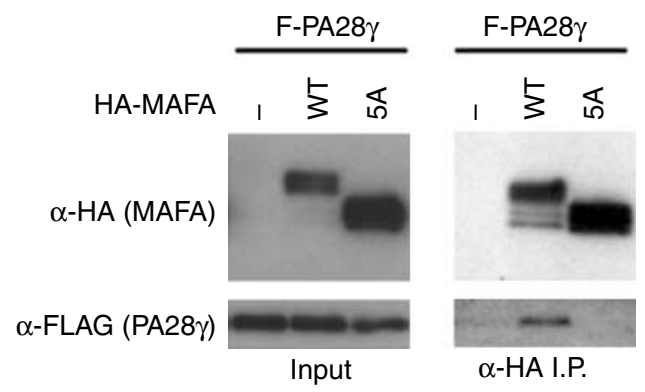

Figure 4 Interaction between HA-MAFA and F-PA28 $\gamma$ was analyzed using an immunoprecipitation assay. HeLa cells were transfected with an expression plasmid for F-PA28 $\gamma$ together with an empty vector or an expression plasmid for HA-MAFA (WT or $5 A)$. To avoid MAFA degradation, cells were treated with the proteasome inhibitors MG132 $(5 \mu \mathrm{M})$ for $8 \mathrm{~h}$ before harvest. Cell extracts were subjected to immunoprecipitation with an anti-HA antibody, and the immune complexes ( $\alpha$-HA I.P., right panels) were examined by immunoblot analysis using an anti-HA or antiFLAG antibody. As a control, the total cell extract (input, left panels) was also analyzed.

not significantly affected by the presence of F-PA28 $\gamma$. The reduction of endogenous PA28 $\gamma$ by shRNA resulted in a significant increase in the half-life of WT HA-MAFA protein (Fig. 3D). These results demonstrated that PA28 $\gamma$ promoted proteasomal degradation of MAFA that depended on its phosphorylation status.

\section{PA28 $\gamma$ associates with MAFA depending on phosphorylation}

The interaction between MAFA and PA28 $\gamma$ was examined by the co-expression of WT or 5A HA-MAFA and F-PA28 $\gamma$ in HeLa cells. Cell extracts were subjected to immunoprecipitation with an anti-HA antibody and immunoblot analysis with anti-HA and antiFLAG antibodies (Fig. 4). In the context of WT HA-MAFA co-expression, F-PA28 $\gamma$ was detected in the
anti-HA immunoprecipitate. However, the MAFA 5A mutant did not co-immunoprecipitate F-PA28 $\gamma$. Thus, MAFA interacted with PA28 $\gamma$ in a phosphorylation-dependent manner.

\section{GSK3 $\beta$ cooperates with PA28 $\gamma$ to induce MAFA degradation}

We previously demonstrated that MAFA is first phosphorylated at Ser65 by an unidentified kinase and is then sequentially phosphorylated at Ser61, Thr57, Thr53, and Ser49 by GSK3 (Han et al. 2007). We thus investigated the role of GSK3 in PA28 $\gamma$ mediated degradation of MAFA. Treatment of the transfected cells with SB216763, a specific inhibitor of GSK3, induced the accumulation of hypo-phosphorylated forms of HA-MAFA and blunted the PA28 $\gamma$-mediated degradation of MAFA (Fig. 5A). This result indicated that the phosphorylation of MAFA by GSK3 is required for PA28 $\gamma$-mediated protein degradation.

Then, NIH3T3 cells were transfected with a constant amount of the HA-MAFA expression plasmid and an increasing amount of the F-PA28 $\gamma$ expression vector. Expression plasmids for WT, constitutively active (CA), or kinase-deficient (KD) forms of GSK3 $\beta$ were also included. Cell extracts were analyzed for protein expression by immunoblotting (Fig. 5B). In this experimental setting, the amount of the PA28 $\gamma$ expression vector was kept low so that PA28 $\gamma$ alone promote only marginal degradation of WT MAFA. However, co-expression of GSK3 $\beta$, particularly the CA form, enhanced MAFA degradation by PA28 $\gamma$. In contrast, the $\mathrm{KD}$ form of GSK3 $\beta$ had little effect, indicating that the stimulation of PA28 $\gamma$-mediated MAFA degradation by GSK3 $\beta$ depends on its kinase activity. The amount of the 5A form of MAFA was not largely affected by co-expression of F-PA28 $\gamma$ or either form of GSK3 $\beta$.

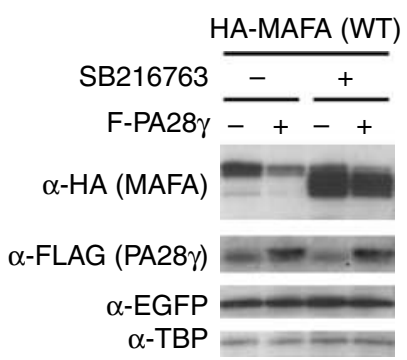

B

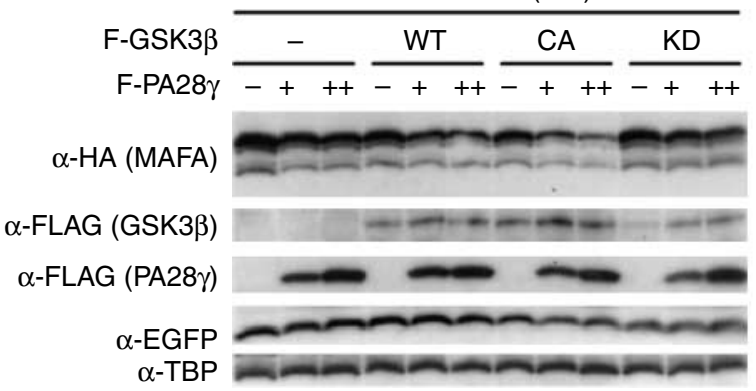

HA-MAFA (5A)

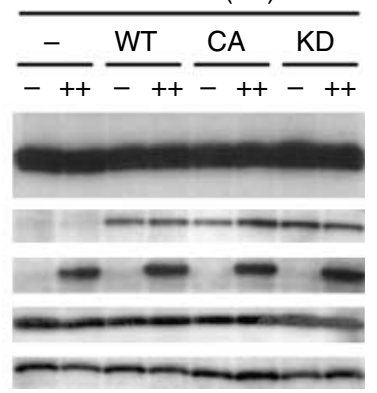

Figure 5 GSK3 cooperates with PA28 $\gamma$ to induce the degradation of MAFA. (A) In1024 cells transfected with the indicated expression plasmids were treated with the GSK3 inhibitor SB216763 $(20 \mu \mathrm{M})$ for $15 \mathrm{~h}$. The cell extracts were analyzed by immunoblot using the indicated antibodies. (B) NIH3T3 cells were transfected with a constant amount of expression plasmids for HA-MAFA (0.3 $\mu$ g, WT or $5 A)$ and F-GSK3 $\beta(0.1 \mu \mathrm{g}, \mathrm{WT}$, constitutively active (CA, S9A) or kinase deficient (KD, K85R)), together with an increasing amount of F-PA28 $\gamma(+, 0.2 \mu \mathrm{g} ;++, 0.4 \mu \mathrm{g})$. Cell extracts were examined by immunoblot analysis using the indicated antibodies. 
A

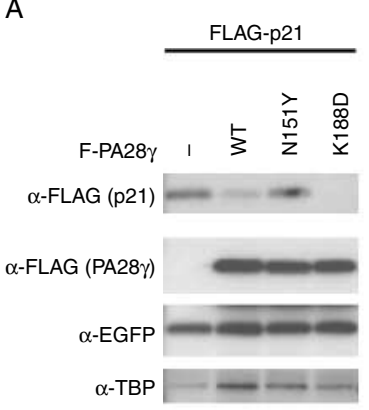

$B$
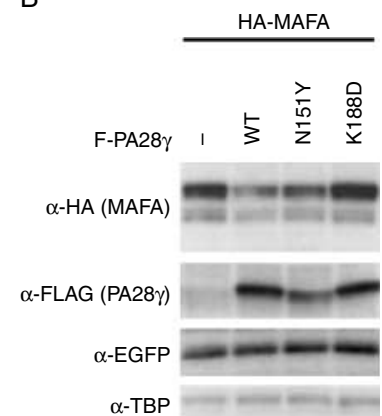

Figure 6 Differential effects of PA28 $\gamma$ mutants on the degradation of p21 and MAFA. (A and B) NIH3T3 cells were transfected with $0.2 \mu \mathrm{g}$ of an expression plasmid for FLAG-p21 (A) or HA-MAFA

(B) together with an expression vector $(0.6 \mu \mathrm{g})$ for wild-type (WT) or mutant (N151Y and K188D) F-PA28 $\gamma$. Cell extracts were examined by immunoblot analysis with anti-HA and anti-FLAG antibodies. A constant amount $(0 \cdot 1 \mu \mathrm{g})$ of an EGFP expression plasmid was included to monitor transfection efficiency and TBP was analyzed as a loading control.

These results indicate that GSK3 $\beta$ cooperates with PA28 $\gamma$ to induce the degradation of MAFA through the phosphorylation of its target serine and threonine residues.

\section{PA28 $\gamma$-mediated degradation of MAFA and p21 occurs through distinct molecular mechanisms}

To investigate the molecular mechanism by which PA28 $\gamma$ stimulates MAFA protein degradation, we utilized two well-defined PA28 $\gamma$ mutants. It has been proposed that the PA28 $\gamma$ heptamer binds to the 20S proteasome and stimulates its trypsin-like activity (Realini et al. 1997). The N151Y mutant is capable of binding to the 20S proteasome but is unable to enhance its catalytic activity (Zhang et al. 1998). In contrast, the K188D mutant is hyperactive, activating not only the $20 \mathrm{~S}$ proteasome trypsin-like activity but also its chymotrypsin-like and post-glutamyl peptidyl hydrolyzing activities (Li et al. 2001).

To confirm the characteristics of these mutants, we tested their ability to stimulate degradation of the p21/CIP1, a previously identified PA28 $\gamma$ target (Chen et al. 2007, Li et al. 2007). As expected, p21 degradation was enhanced by WT PA28 $\gamma$ but not by the N151Y mutant (Fig. 6A), whereas the K188D mutant more potently induced p21 degradation. We then tested the effect of these mutants on MAFA stability (Fig. 6B). The defective N151Y and the WT PA28 $\gamma$ induced MAFA degradation to a similar extent, whereas the hyperactive mutant K188D did not induce MAFA degradation. These results indicated that PA28 $\gamma$ stimulates the degradation of p21 and MAFA by distinct molecular mechanisms.

\section{Discussion}

Previous studies have shown that the phosphorylation of MAFA at multiple sites (Ser49, Thr53, Thr57, Ser61, and Ser65) by a priming kinase and GSK3 are critical for its proteasomal degradation (Han et al. 2007, Rocques et al. 2007), but the molecular mechanism underlying this degradation was unknown. In this study, several lines of evidence suggest that PA28 $\gamma$ is involved in the degradation of MAFA. Overexpression of PA28 $\gamma$ stimulated the degradation of MAFA depending on the phosphorylation and attenuated transactivation of the insulin promoter. Furthermore, GSK3 cooperated with PA28 $\gamma$ to enhance MAFA degradation. Conversely, the knockdown of PA28 $\gamma$ using siRNA or shRNA increased endogenous and exogenous MAFA protein, while pharmacological inhibition of GSK3 eliminated the PA28 $\gamma$-mediated degradation of MAFA. Co-transfection and co-immunoprecipitation experiments revealed that PA28 $\gamma$ binding to MAFA was dependent on phosphorylation.

Previous reports have demonstrated that PA28 $\gamma$ directly binds to p21 and SRC3 and stimulates their degradation via the $20 \mathrm{~S}$ proteasome by an unknown mechanism that is not dependent on poly-ubiquitination and the 19S regulatory subunit (Chen et al. 2007, $\mathrm{Li}$ et al. 2007). Using two well-characterized PA28 $\gamma$ mutants, N151Y and K188D, we showed that PA28r stimulates the degradation of MAFA and p21 by distinct molecular mechanisms. The N151Y mutant can bind to the $20 \mathrm{~S}$ proteasome but is unable to enhance its protease activity. Here, the N151Y mutant did not stimulate p21 degradation but it enhanced MAFA degradation. In contrast, the 'hyperactive' K188D mutant stimulated p21 degradation more than WT PA28 $\gamma$ but it did not enhance MAFA degradation. Additional studies are required to elucidate the exact molecular mechanism to explain how PA28 $\gamma$ enhances MAFA degradation.

PA28 $\gamma$ knockout mice exhibit growth retardation, and embryonic fibroblasts derived from them have defects in mitosis and apoptosis (Murata et al. 1999). Thus, it has been proposed that p21 and p53 are potential targets of PA28 $\gamma$ in the regulation of cell cycle progression and apoptosis (Chen et al. 2007, Li et al. 2007, Zhang \& Zhang 2008), but the exact role of PA28 $\gamma$ in the cell cycle and other biological processes is largely unknown. In this study, MAFA was identified as a new target of PA28 $\gamma$. Examination of the development and function of pancreatic $\beta$-cells in PA28 $\gamma$-knockout mice is warranted to determine whether PA28 $\gamma$ is involved in $\beta$-cell morphology and function. As MAFA regulates cell differentiation as well as oncogenic transformation (Nishizawa et al. 2003, Pouponnot et al. 2006), PA28 $\gamma$ may also be involved in these processes. Further studies of PA28 $\gamma$ and its 
target proteins will help define its role in the degradation of a subset of nuclear proteins important in a variety of biological processes.

\section{Declaration of interest}

The authors declare that there is no conflict of interest that could be perceived as prejudicing the impartiality of the research reported.

\section{Funding}

This work was supported by Grants-in-Aid for Scientific Research on Priority Areas (DECODE), for Scientific Research (C), the Global COE Program in NAIST from MEXT, Japan, a grant from the Mitsubishi Foundation, and a grant from the Takeda Science Foundation to K K.

\section{References}

Aramata S, Han SI, Yasuda K \& Kataoka K 2005 Synergistic activation of the insulin gene promoter by the beta-cell enriched transcription factors MafA, Beta2, and Pdx1. Biochimica et Biophysica Acta 1730 41-46. (doi:10.1016/j.bbaexp.2005.05.009)

Artner I, Blanchi B, Raum JC, Guo M, Kaneko T, Cordes S, Sieweke M \& Stein R 2007 MafB is required for islet beta cell maturation. PNAS 104 3853-3858. (doi:10.1073/pnas.0700013104)

Benkhelifa S, Provot S, Lecoq O, Pouponnot C, Calothy G \& FelderSchmittbuhl MP 1998 mafA, a novel member of the maf protooncogene family, displays developmental regulation and mitogenic capacity in avian neuroretina cells. Oncogene 17 247-254. (doi:10. 1038/sj.onc.1201898)

Benkhelifa S, Provot S, Nabais E, Eychene A, Calothy G \& FelderSchmittbuhl MP 2001 Phosphorylation of MafA is essential for its transcriptional and biological properties. Molecular and Cellular Biology 21 4441-4452. (doi:10.1128/MCB.21.14.4441-4452.2001)

Chen X, Barton LF, Chi Y, Clurman BE \& Roberts JM 2007 Ubiquitinindependent degradation of cell-cycle inhibitors by the REGgamma proteasome. Molecular Cell 26 843-852. (doi:10.1016/j.molcel.2007. 05.022)

Docherty HM, Hay CW, Ferguson LA, Barrow J, Durward E \& Docherty K 2005 Relative contribution of PDX-1, MafA and E47/beta2 to the regulation of the human insulin promoter. Biochemical Journal 389 813-820. (doi:10.1042/BJ20041891)

Dubiel W, Pratt G, Ferrell K \& Rechsteiner M 1992 Purification of an $11 \mathrm{~S}$ regulator of the multicatalytic protease. Journal of Biological Chemistry 267 22369-22377.

Forster A, Masters EI, Whitby FG, Robinson H \& Hill CP 2005 The 1.9 A structure of a proteasome-11S activator complex and implications for proteasome-PAN/PA700 interactions. Molecular Cell 18 589-599. (doi:10.1016/j.molcel.2005.04.016)

Han SI, Aramata S, Yasuda K \& Kataoka K 2007 MafA stability in pancreatic beta cells is regulated by glucose and is dependent on its constitutive phosphorylation at multiple sites by glycogen synthase kinase 3. Molecular and Cellular Biology 27 6593-6605. (doi:10.1128/ MCB.01573-06)

Ho SN, Hunt HD, Horton RM, Pullen JK \& Pease LR 1989 Sitedirected mutagenesis by overlap extension using the polymerase chain reaction. Gene 77 51-59. (doi:10.1016/0378-1119(89) 90358-2)

Kajihara M, Sone H, Amemiya M, Katoh Y, Isogai M, Shimano H, Yamada N \& Takahashi S 2003 Mouse MafA, homologue of zebrafish somite Maf 1, contributes to the specific transcriptional activity through the insulin promoter. Biochemical and Biophysical Research Communications 312 831-842. (doi:10.1016/j.bbrc.2003. 10.196)

Kanai K, Reza HM, Kamitani A, Hamazaki Y, Han SI, Yasuda K \& Kataoka K 2010 SUMOylation negatively regulates transcriptional and oncogenic activities of MafA. Genes to Cells 15 971-982. (doi:10. $1111 / \mathrm{j} .1365-2443.2010 .01431 . x)$

Kaneto H, Matsuoka TA, Nakatani Y, Miyatsuka T, Matsuhisa M, Hori M \& Yamasaki Y 2005 A crucial role of MafA as a novel therapeutic target for diabetes. Journal of Biological Chemistry 280 15047-15052. (doi:10.1074/jbc.M412013200)

Kataoka K, Yoshitomo-Nakagawa K, Shioda S \& Nishizawa M 2001 A set of Hox proteins interact with the Maf oncoprotein to inhibit its DNA binding, transactivation, and transforming activities. Journal of Biological Chemistry 276 819-826. (doi:10.1074/jbc.M007643200)

Kataoka K, Han SI, Shioda S, Hirai M, Nishizawa M \& Handa H 2002 MafA is a glucose-regulated and pancreatic beta-cell-specific transcriptional activator for the insulin gene. Journal of Biological Chemistry 277 49903-49910. (doi:10.1074/jbc.M206796200)

Kataoka K, Shioda S, Ando K, Sakagami K, Handa H \& Yasuda K 2004 Differentially expressed Maf family transcription factors, c-Maf and MafA, activate glucagon and insulin gene expression in pancreatic islet alpha- and beta-cells. Journal of Molecular Endocrinology 32 9-20. (doi:10.1677/jme.0.0320009)

Kato T, Shimano H, Yamamoto T, Yokoo T, Endo Y, Ishikawa M, Matsuzaka T, Nakagawa Y, Kumadaki S, Yahagi N et al. 2006 Granuphilin is activated by SREBP-1c and involved in impaired insulin secretion in diabetic mice. Cell Metabolism 4 143-154. (doi:10.1016/j.cmet.2006.06.009)

Li J, Gao X, Ortega J, Nazif T, Joss L, Bogyo M, Steven AC \& Rechsteiner M 2001 Lysine 188 substitutions convert the pattern of proteasome activation by REGgamma to that of REGs alpha and beta. EMBO Journal 20 3359-3369. (doi:10.1093/emboj/20.13. 3359)

Li X, Lonard DM, Jung SY, Malovannaya A, Feng Q, Qin J, Tsai SY, Tsai MJ \& O'Malley BW 2006 The SRC-3/AIB1 coactivator is degraded in a ubiquitin- and ATP-independent manner by the REGgamma proteasome. Cell 124 381-392. (doi:10.1016/j.cell. 2005.11.037)

Li X, Amazit L, Long W, Lonard DM, Monaco JJ \& O'Malley BW 2007 Ubiquitin- and ATP-independent proteolytic turnover of p21 by the REGgamma-proteasome pathway. Molecular Cell 26 831-842. (doi:10.1016/j.molcel.2007.05.028)

Ma CP, Slaughter CA \& DeMartino GN 1992 Identification, purification, and characterization of a protein activator (PA28) of the $20 \mathrm{~S}$ proteasome (macropain). Journal of Biological Chemistry 267 10515-10523.

Matsuoka TA, Zhao L, Artner I, Jarrett HW, Friedman D, Means A \& Stein R 2003 Members of the large Maf transcription family regulate insulin gene transcription in islet beta cells. Molecular and Cellular Biology 23 6049-6062. (doi:10.1128/MCB.23.17.60496062.2003)

Moriishi K, Okabayashi T, Nakai K, Moriya K, Koike K, Murata S, Chiba T, Tanaka K, Suzuki R, Suzuki T et al. 2003 Proteasome activator PA28gamma-dependent nuclear retention and degradation of hepatitis C virus core protein. Journal of Virology 77 10237-10249. (doi:10.1128/JVI.77.19.10237-10249.2003)

Murata S, Kawahara H, Tohma S, Yamamoto K, Kasahara M, Nabeshima Y, Tanaka K \& Chiba T 1999 Growth retardation in mice lacking the proteasome activator PA28gamma. Journal of Biological Chemistry 274 38211-38215. (doi:10.1074/jbc.274.53. 38211)

Nishimura W, Kondo T, Salameh T, El Khattabi I, Dodge R, BonnerWeir S \& Sharma A 2006 A switch from MafB to MafA expression accompanies differentiation to pancreatic beta-cells. Developmental Biology 293 526-539. (doi:10.1016/j.ydbio.2006.02.028) 
Nishimura W, Rowan S, Salameh T, Maas RL, Bonner-Weir S, Sell SM \& Sharma A 2008 Preferential reduction of beta cells derived from Pax6-MafB pathway in MafB deficient mice. Developmental Biology 314 443-456. (doi:10.1016/j.ydbio.2007.12.009)

Nishizawa M, Kataoka K \& Vogt PK 2003 MafA has strong cell transforming ability but is a weak transactivator. Oncogene 22 7882-7890. (doi:10.1038/sj.onc.1206526)

Noso S, Kataoka K, Kawabata Y, Babaya N, Hiromine Y, Yamaji K, Fujisawa T, Aramata S, Kudo T, Takahashi S et al. 2010 Insulin transactivator MafA regulates intrathymic expression of insulin and affects susceptibility to type 1 diabetes. Diabetes $\mathbf{5 9} 2579-2587$. (doi:10.2337/db10-0476)

Ochi H, Ogino H, Kageyama Y \& Yasuda K 2003 The stability of the lens-specific Maf protein is regulated by fibroblast growth factor (FGF)/ERK signaling in lens fiber differentiation. Journal of Biological Chemistry 278 537-544. (doi:10.1074/jbc.M208380200)

Ogino H \& Yasuda K 1998 Induction of lens differentiation by activation of a bZIP transcription factor, L-Maf. Science 280 115-118. (doi:10.1126/science.280.5360.115)

Olbrot M, Rud J, Moss LG \& Sharma A 2002 Identification of beta-cellspecific insulin gene transcription factor RIPE3b1 as mammalian MafA. PNAS 99 6737-6742. (doi:10.1073/pnas.102168499)

Pouponnot C, Sii-Felice K, Hmitou I, Rocques N, Lecoin L, Druillennec S, Felder-Schmittbuhl MP \& Eychene A 2006 Cell context reveals a dual role for Maf in oncogenesis. Oncogene $\mathbf{2 5}$ 1299-1310. (doi:10.1038/sj.onc.1209171)

Realini C, Jensen CC, Zhang Z, Johnston SC, Knowlton JR, Hill CP \& Rechsteiner M 1997 Characterization of recombinant REGalpha, REGbeta, and REGgamma proteasome activators. Journal of Biological Chemistry 272 25483-25492. (doi:10.1074/jbc. 272.41.25483)

Reza HM, Ogino H \& Yasuda K 2002 L-Maf, a downstream target of Pax6, is essential for chick lens development. Mechanisms of Development 116 61-73. (doi:10.1016/S0925-4773(02)00137-5)
Rocques N, Abou Zeid N, Sii-Felice K, Lecoin L, Felder-Schmittbuhl MP, Eychene A \& Pouponnot C 2007 GSK-3-mediated phosphorylation enhances Maf-transforming activity. Molecular Cell 28 584-597. (doi:10.1016/j.molcel.2007.11.009)

Sii-Felice K, Pouponnot C, Gillet S, Lecoin L, Girault JA, Eychene A \& Felder-Schmittbuhl MP 2005 MafA transcription factor is phosphorylated by p38 MAP kinase. FEBS Letters 579 3547-3554. (doi:10. 1016/j.febslet.2005.04.086)

Wang H, Brun T, Kataoka K, Sharma AJ \& Wollheim CB 2007 MAFA controls genes implicated in insulin biosynthesis and secretion. Diabetologia 50 348-358. (doi:10.1007/s00125-006-0490-2)

Whitby FG, Masters EI, Kramer L, Knowlton JR, Yao Y, Wang CC \& Hill CP 2000 Structural basis for the activation of $20 \mathrm{~S}$ proteasomes by $11 S$ regulators. Nature 408 115-120. (doi:10.1038/35040607)

Wojcik C, Tanaka K, Paweletz N, Naab U \& Wilk S 1998 Proteasome activator (PA28) subunits, alpha, beta and gamma (Ki antigen) in NT2 neuronal precursor cells and HeLa S3 cells. European Journal of Cell Biology 77 151-160.

Zhang Z \& Zhang R 2008 Proteasome activator PA28 gamma regulates p53 by enhancing its MDM2-mediated degradation. EMBO Journal 27 852-864. (doi:10.1038/emboj.2008.25)

Zhang Z, Clawson A, Realini C, Jensen CC, Knowlton JR, Hill CP \& Rechsteiner M 1998 Identification of an activation region in the proteasome activator REGalpha. PNAS 95 2807-2811. (doi:10. 1073/pnas.95.6.2807)

Zhang C, Moriguchi T, Kajihara M, Esaki R, Harada A, Shimohata H, Oishi H, Hamada M, Morito N, Hasegawa K et al. 2005 MafA is a key regulator of glucose-stimulated insulin secretion. Molecular and Cellular Biology 25 4969-4976. (doi:10.1128/MCB.25.12.4969-4976. 2005)

Received in final form 7 May 2011

Accepted 6 June 2011

Made available online as an Accepted Preprint 6 June 2011 